\title{
Dificuldades diagnósticas no osteoma osteoide do cotovelo: estudo clínico, radiológico e histopatológico*
}

\author{
Diagnostic difficulties in osteoid osteoma of the elbow: clinical, radiological and histopathological study \\ Ana Cristina Cotta ${ }^{1}$, Rogério Teles de Melo $^{2}$, Renato César Rezende de Castro ${ }^{3}$, Francineide Sadala \\ de Souza ${ }^{4}$, Yana Senna Jeronymo Najjar ${ }^{5}$, Júlia Filardi de Oliveira Paim ${ }^{1}$, Ricardo Karam Kalil ${ }^{4}$
}

\begin{abstract}
Resumo Objetivo: Descrever os aspectos clínicos e de imagem que podem auxiliar no diagnóstico correto do osteoma osteoide no cotovelo. Materiais e Métodos: Realizado estudo retrospectivo de sete pacientes com diagnóstico de osteoma osteoide no cotovelo confirmado histologicamente por biópsia óssea. Eles tiveram seus prontuários médicos e exames de imagem revisados. Resultados: Do total de 142 pacientes identificados em nossos arquivos, 4,9\% apresentavam a lesão no cotovelo, com predomínio no sexo masculino $(2,5: 1)$ e idade média de 25 anos. Dor e limitação de movimento foram os sintomas mais comuns. Cinco pacientes $(71,4 \%)$ receberam outro diagnóstico clínico prévio. A duração média dos sintomas foi de 21 meses. As radiografias não demonstraram o nidus em 42,8\% dos casos. A tomografia computadorizada e a ressonância magnética mostraram claramente o nidus. Derrame articular foi um achado constante. $\mathrm{O}$ aspecto histológico observado foi o usual. A ressecção cirúrgica promoveu alívio dos sintomas e/ou melhora funcional em todos os casos. Conclusão: É importante considerar a possibilidade de osteoma osteoide em paciente adulto jovem com dor, limitação do movimento e sinais de sinovite no cotovelo, refratária ao tratamento conservador. Esclerose óssea, espessamento cortical e/ou reação periosteal detectados na radiografia permitem direcionar a tomografia computadorizada para a visualização precisa do nidus.
\end{abstract}

Unitermos: Osteoma osteoide; Intra-articular; Cotovelo; Sinovite; Imagem; Biópsia.

\begin{abstract}
Objective: To describe the clinical and imaging findings that may aid in correctly diagnosing osteoid osteoma of the elbow. Materials and Methods: The authors have retrospectively evaluated seven patients diagnosed with osteoid osteoma of the elbow histologically confirmed by bone biopsy. The patients' medical records and imaging studies were reviewed. Results: Among the 142 patients with osteoid osteoma identified in the archives of the institutions, 4.9\% had the lesion in the elbow, predominantly in men (2.5:1), with a mean age of 25 years. Pain and limitation of movement were the most common symptoms. Five of the patients (71.4\%) had previously received a different clinical diagnosis. The mean duration of symptoms was 21 months. Radiography did not demonstrate the nidus in $42.8 \%$ of cases. Computed tomography and magnetic resonance imaging clearly demonstrated the nidus. Joint effusion was a constant finding. The histological findings corresponded to the ones usually observed in such cases. Surgical resection has brought relief of symptoms and/or functional improvement in all of the cases. Conclusion: It is important to consider the possibility of osteoid osteoma in young adult patients with pain, limitation of movement and signs of synovitis in the elbow refractory to conservative treatment. The detection of bone sclerosis, cortical thickening and/or periosteal reaction at radiography allows directing computed tomography towards the accurate identification of the nidus.
\end{abstract}

Keywords: Osteoid osteoma; Intra-articular; Elbow; Synovitis; Imaging; Biopsy.

Cotta AC, Melo RT, Castro RCR, Souza FS, Najjar YSJ, Paim JFO, Kalil RK. Dificuldades diagnósticas no osteoma osteoide do cotovelo: estudo clínico, radiológico e histopatológico. Radiol Bras. 2012 Jan/Fev;45(1):13-19.

* Trabalho realizado nos Departamentos de Patologia Cirúrgica, Radiologia e Ortopedia da Rede Sarah de Hospitais de Reabilitação, Hospitais Sarah Belo Horizonte, MG, e Sarah Brasília, DF, Brasil.

1. Especialistas em Anatomia Patológica, Médicas Patologistas Cirúrgicas do Hospital Sarah, Belo Horizonte, MG, Brasil.

2. Membro Titular do Colégio Brasileiro de Radiologia e Diagnóstico por Imagem (CBR), Médico Radiologista do Hospital Sarah, Belo Horizonte, MG, Brasil.

3. Especialista em Ortopedia e Traumatologia, Médico Ortopedista do Hospital Sarah, Belo Horizonte, MG, Brasil.

4. Especialistas em Anatomia Patológica, Médicos Patologistas Cirúrgicos do Hospital Sarah, Brasília, DF, Brasil.

5. Membro Titular do Colégio Brasileiro de Radiologia e Diagnóstico por Imagem (CBR), Médica Radiologista do Hospital Sarah, Brasília, DF, Brasil.

\section{INTRODUÇÃO}

O osteoma osteoide é um tumor ósseo benigno, pequeno e relativamente comum, que possui características clínicas, radiológicas e patológicas bem definidas ${ }^{(1-3)}$. É mais prevalente na faixa etária de 10 a 25

Endereço para correspondência: Dr. Rogério Teles de Melo. Avenida Amazonas, 5953, Gameleira. Belo Horizonte, MG, Brasil, 30510-000. E-mail: rogeriomelo@sarah.br

Recebido para publicação em 6/11/2011. Aceito, após revisão, em 11/1/2012. anos. A maioria dos pacientes apresenta o sintoma clássico de dor óssea, que piora à noite e melhora com uso de aspirina ou outro anti-inflamatório não esteroide. A localização mais comum é a diáfise ou metáfise dos ossos longos, sobretudo no fêmur e na tíbia.

Quando ocorre em localização intra-articular, as manifestações clínicas são inespecíficas e as alterações radiográficas podem ser sutis, representando um desafio diagnóstico. Pacientes com osteoma os- 
teoide localizado no cotovelo frequentemente cursam com dor, sinovite crônica, derrame articular, limitação do movimento e contratura em flexão, simulando uma artropatia inflamatória. A esclerose reacional nas radiografias pode ser mínima ou ausente, levando a um atraso significativo no diagnóstico ou a um diagnóstico equivocado $^{(4-6)}$.

O objetivo deste trabalho é ressaltar as características clínicas e radiológicas que permitem o diagnóstico precoce e conduta adequada nos casos de osteoma osteoide localizado no cotovelo.

\section{MATERIAIS E MÉTODOS}

Na base de dados dos Hospitais Sarah Belo Horizonte e Sarah Brasília, foram identificados 142 pacientes com diagnóstico de osteoma osteoide com confirmação histológica. Os sete pacientes que apresentavam a lesão no cotovelo foram selecionados e tiveram seus prontuários e exames de imagem revisados.

As informações clínicas coletadas foram sexo, idade, lado acometido, presença de dor, febre, edema, limitação funcional, duração dos sintomas, diagnósticos e tratamentos prévios, resposta ao uso de anti-inflamatórios não esteroides e ao tratamento cirúrgico realizado (Tabela 1).

Todos os sete pacientes foram submetidos a exames de radiografia simples e tomografia computadorizada (TC). Três pacientes realizaram também exame de ressonância magnética (RM) em equipamento de 1,5 tesla. As radiografias foram utilizadas para avaliar a presença do nidus, halo de esclerose, espessamento cortical e periostite. Na TC, foram analisados a aparência e localização do nidus, sinais de es- clerose, espessamento e ruptura da cortical, além da presença de derrame articular. As imagens de RM foram úteis para avaliar a alteração na intensidade de sinal da medular óssea, a identificação do nidus, presença de derrame articular, sinovite e anormalidades em tecidos moles adjacentes. Os achados de imagem estão descritos na Tabela 2 .

O estudo foi aprovado pelo Comitê de Ética em Pesquisa da Instituição.

\section{RESULTADOS}

Do total de 142 pacientes com diagnóstico de osteoma osteoide, sete $(4,9 \%)$ apresentavam a lesão no cotovelo, sendo cinco pacientes do sexo masculino e dois do sexo feminino (relação de 2,5:1), com idades variando entre 11 e 47 anos (média de 25 anos). O úmero distal foi o local acometido

Tabela 1 Apresentação clínica e controle.

\begin{tabular}{|c|c|c|c|c|c|c|c|c|}
\hline $\begin{array}{c}\text { Caso } \\
n^{\circ}\end{array}$ & Sexo & $\begin{array}{l}\text { Idade } \\
\text { (anos) }\end{array}$ & Lado & Sintomas & $\begin{array}{l}\text { Duração dos } \\
\text { sintomas } \\
\text { (meses) }\end{array}$ & $\begin{array}{l}\text { Diagnóstico } \\
\text { prévio }\end{array}$ & Tratamento prévio & $\begin{array}{c}\text { Controle } \\
\text { pós-operatório }\end{array}$ \\
\hline 1 & M & 21 & $E$ & Dor e perda do movimento & 12 & Pseudogota & Fisioterapia & Alívio da dor \\
\hline 2 & M & 20 & $\mathrm{D}$ & Dor e perda da extensão & 6 & - & - & $\begin{array}{l}\text { Alívio da dor; me- } \\
\text { Ihora funcional }\end{array}$ \\
\hline 3 & $\mathrm{~F}$ & 15 & $E$ & $\begin{array}{c}\text { Dificuldade em realizar extensão } \\
\text { e dor }\end{array}$ & 36 & Osteomielite & Antibiótico & Alívio da dor \\
\hline 4 & M & 11 & $E$ & $\begin{array}{l}\text { Dor, calor e edema; febre inicial; } \\
\text { limitação funcional }\end{array}$ & 4 & Tendinite & Fisioterapia & Alívio da dor \\
\hline 5 & M & 18 & $\mathrm{D}$ & $\begin{array}{c}\text { Dor contínua e incapacitante } \\
\text { que piora à noite, irradiando } \\
\text { para o punho }\end{array}$ & 24 & Tendinite & $\begin{array}{l}\text { Anti-inflamatório não } \\
\text { esteroide, sem alívio; } \\
\text { repouso }\end{array}$ & Melhora funcional \\
\hline 6 & M & 47 & $E$ & $\begin{array}{c}\text { Dor; perda da força no braço, } \\
\text { antebraço e dedos; fraqueza no } \\
\text { punho e na extensão }\end{array}$ & 48 & Tendinite & $\begin{array}{l}\text { Anti-inflamatório não } \\
\text { esteroide; fisioterapia }\end{array}$ & Alívio da dor \\
\hline 7 & $\mathrm{~F}$ & 47 & $\mathrm{D}$ & $\begin{array}{l}\text { Dor diurna e noturna constante } \\
\text { no epicôndilo medial; nodula- } \\
\text { ção; déficit de movimento }\end{array}$ & 18 & - & $\begin{array}{l}\text { Anti-inflamatório não } \\
\text { esteroide, sem alívio }\end{array}$ & Alívio dos sintomas \\
\hline
\end{tabular}

M, masculino; F, feminino; E, esquerdo; D, direito.

Tabela 2 Achados de imagem relacionados ao osteoma osteoide no cotovelo.

\begin{tabular}{|c|c|c|c|c|c|c|c|}
\hline Caso $n^{\circ}$ & Localização & $\begin{array}{l}\text { Identificação } \\
\text { do nidus (Rx) }\end{array}$ & Aparência do nidus (TC) & $\begin{array}{c}\text { Esclerose } \\
\text { medular (TC) }\end{array}$ & $\begin{array}{l}\text { Espessamento } \\
\text { cortical (Rx e TC) }\end{array}$ & $\begin{array}{l}\text { Ruptura cortical } \\
\text { (TC) }\end{array}$ & $\begin{array}{l}\text { Derrame articular } \\
\text { (TC e RM) }\end{array}$ \\
\hline 1 & Úmero distal & Não & Parcialmente calcificado & Sim & Sim & Sim & Leve \\
\hline 2 & Úmero distal & Não & Densamente calcificado & Sim & Sim & Sim & Acentuado \\
\hline 3 & Úmero distal & Sim & Parcialmente calcificado & Sim & Sim & Sim & Acentuado \\
\hline 4 & Úmero distal & Sim & Parcialmente calcificado & Não & Sim & Sim* & Acentuado \\
\hline 5 & Ulna proximal & Não & $\begin{array}{c}\text { Dois nidus parcialmente } \\
\text { calcificados }\end{array}$ & Não & $\begin{array}{l}\text { Sim (intracortical) } \\
\text { Não (intramedular) }\end{array}$ & $\begin{array}{l}\text { Sim* (intracortical) } \\
\text { Não (intramedular) }\end{array}$ & Não \\
\hline 6 & Ulna proximal & Sim & Densamente calcificado & Não & Sim & $\operatorname{Sim} *$ & Leve \\
\hline 7 & UIna proximal & Não & Não calcificado & Sim & Sim & Não & Leve \\
\hline
\end{tabular}

Rx, radiografia simples; TC, tomografia computadorizada; RM, ressonância magnética. * Componente extraósseo. 
em quatro casos e a ulna proximal em três, sendo quatro casos no cotovelo esquerdo e em três no direito. Dor e limitação de movimento foram os sintomas mais comuns, encontrados em todos os casos ana-

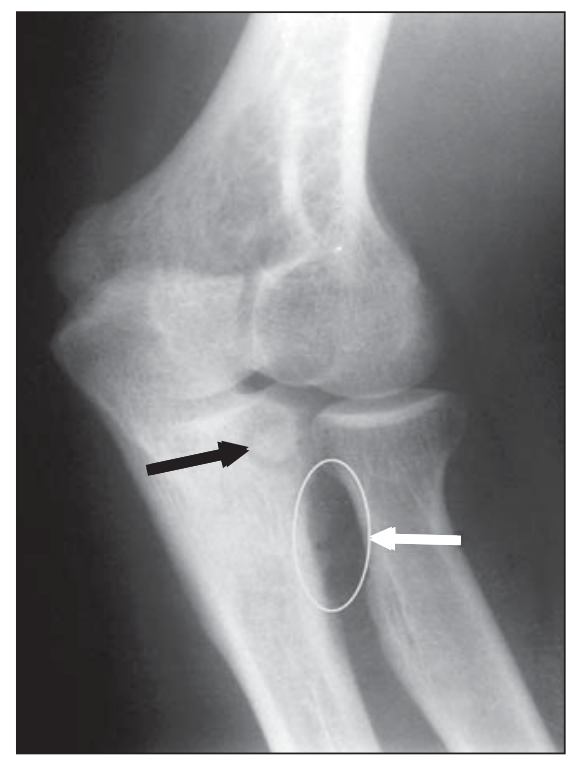

Figura 1. Caso 6. Radiografia simples do cotovelo esquerdo na incidência oblíqua mostra periostite (círculo e seta branca) adjacente ao nidus (seta preta) localizado na ulna proximal.

lisados. Um paciente apresentou também febre, com calor e edema articular.

Dos sete pacientes estudados, cinco $(71,4 \%)$ receberam outro diagnóstico clínico prévio. As hipóteses levantadas antes

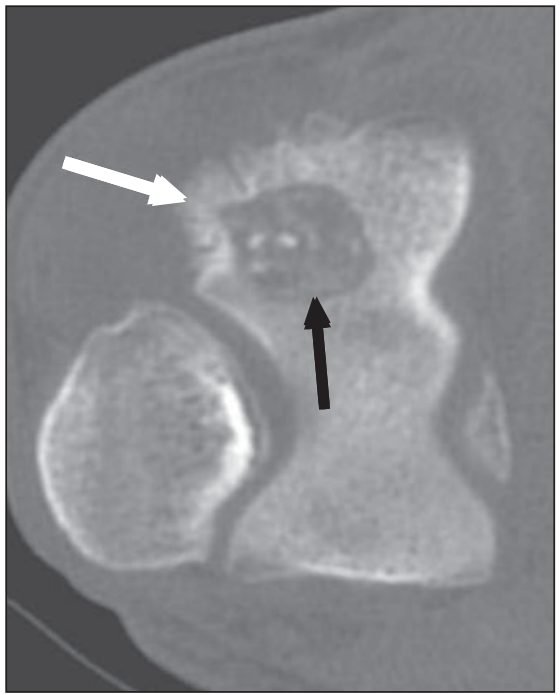

Figura 2. Caso 1. Tomografia no plano axial mostra o nidus osteolítico (seta preta) contendo focos de calcificação no seu interior, associado a periostite (seta branca) na região posterolateral do úmero distal.

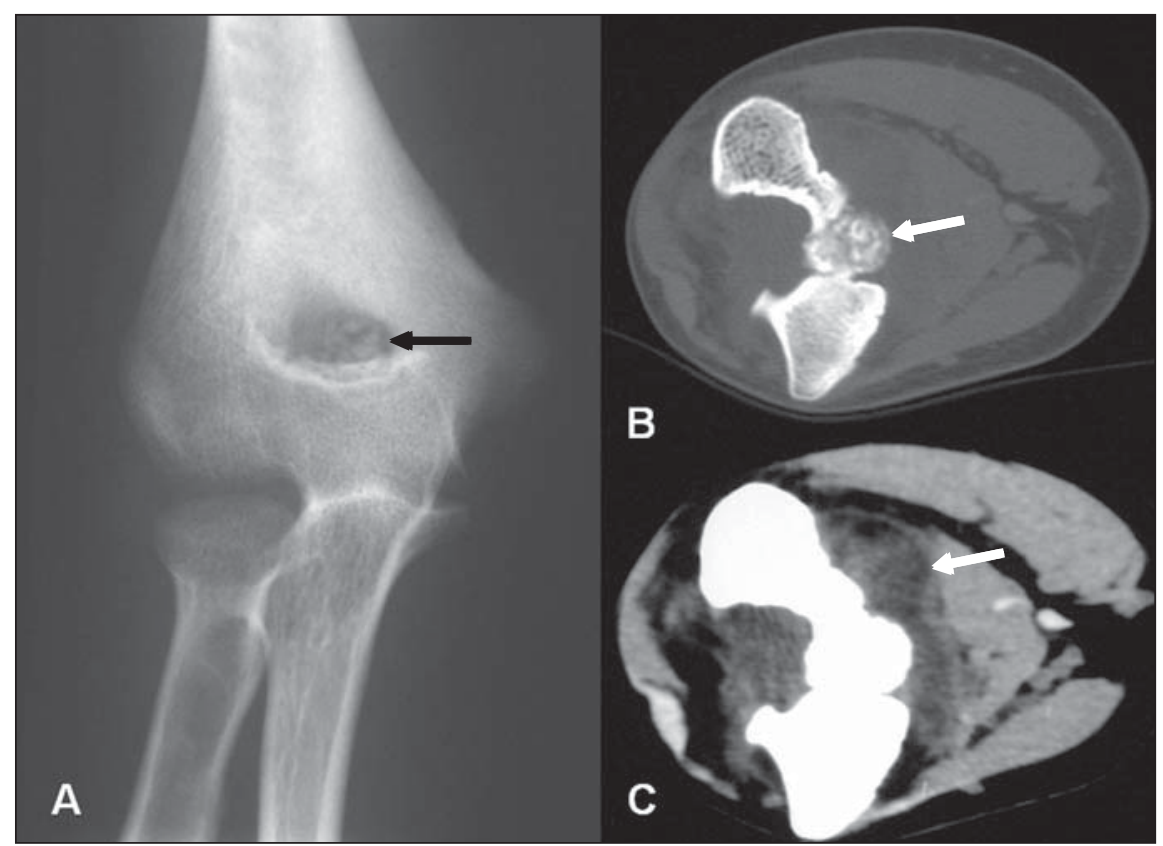

Figura 3. Caso 3. A: Radiografia do cotovelo esquerdo em anteroposterior mostra um nidus (seta) parcialmente calcificado na fossa coronoidea do úmero. B: O corte tomográfico no plano axial com janela óssea mostra o nidus (seta) protruindo na face ventral do úmero. C: Imagem no mesmo plano com janela para partes moles evidencia importante derrame articular associado (seta). do atendimento na nossa Instituição foram: tendinite (três pacientes), pseudogota (um paciente) e osteomielite (um paciente). A duração média dos sintomas foi de 21 meses. O tratamento prévio consistiu no uso de anti-inflamatórios não esteroides em três pacientes, sem alívio dos sintomas em dois deles, fisioterapia em dois pacientes e uso de antibiótico em um caso.

Os exames radiográficos revelaram reação periosteal e/ou espessamento cortical variável em todos os pacientes (Figura 1), mas foram incapazes de demonstrar o $\mathrm{ni}$ dus em três deles $(42,8 \%)$. A TC demonstrou claramente o nidus e a reação óssea em todos os casos analisados (Figura 2). Ruptura da cortical foi identificada em seis dos sete pacientes analisados, sendo que em três deles havia protrusão com componente extraósseo do nidus (Figura 3). Foram identificados dois nidus em um paciente (Figura 4). Em relação ao padrão de mineralização existente dentro dos oito nidus encontrados, a calcificação/ossificação estava ausente em um, era parcial em cinco e densa em dois (Figura 5). A RM, realizada em três pacientes (casos 1, 2 e 4), foi capaz de demonstrar o nidus, derrame articular, sinovite, edema ósseo e em partes moles (Figura 6). $\mathrm{O}$ derrame, presente em seis pacientes, foi considerado leve em três casos e acentuado nos outros três (Figura 3).

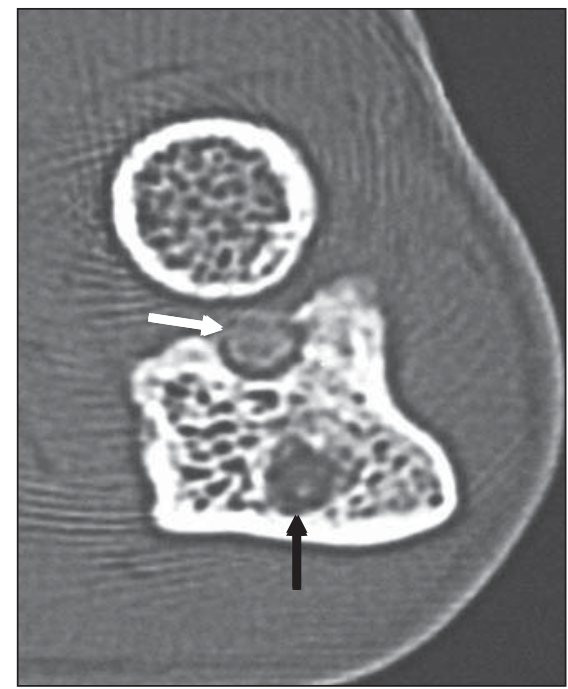

Figura 4. Caso 5. Corte tomográfico no plano axia mostra a presença de dois nidus na ulna proximal, um intramedular (seta preta) e o outro protruindo através de ruptura na cortical (seta branca). Notar a mínima esclerose na medular óssea adjacente às lesões. 

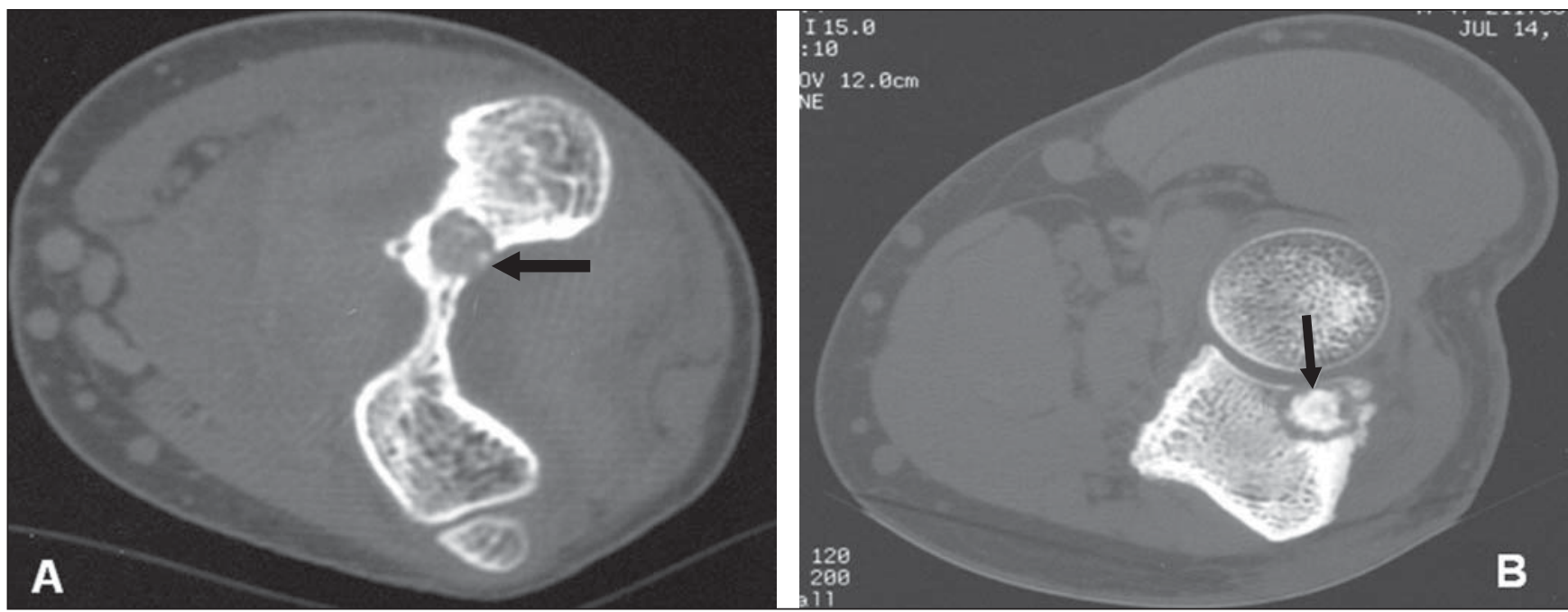

Figura 5. Aparência do nidus na tomografia. A: Lesão osteolítica parcialmente calcificada (seta), menor que 1 cm, localizada junto à fossa do olécrano. B: Nidus localizado na ulna proximal contendo foco central densamente calcificado com halo hipodenso ao seu redor (seta).

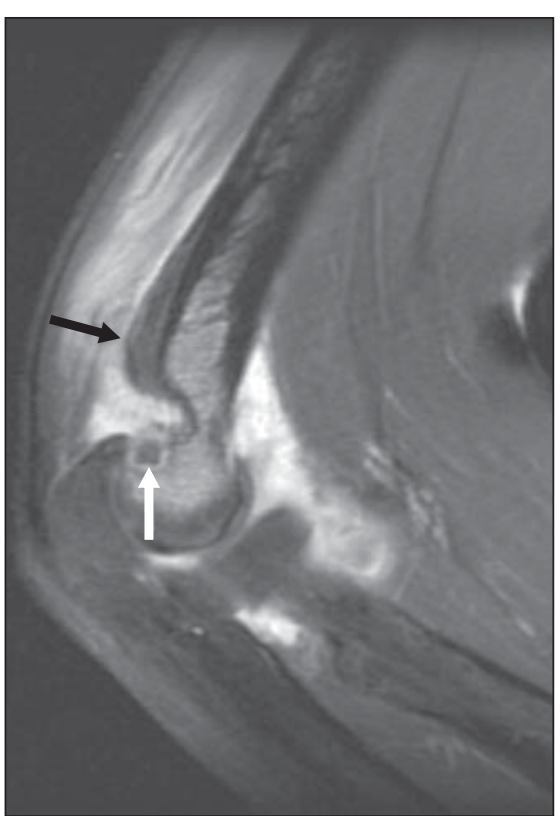

Figura 6. Caso 2. Sagital ponderado em T1 pós contraste com saturação de gordura demonstra o nidus hipointenso (seta branca), o edema na medular óssea e no músculo tríceps, além do importante espessamento e realce sinovial. A reação periosteal sólida (seta preta) na margem posterior da metáfise distal do úmero ocorre distante do nidus porque o periósteo não está presente dentro da cápsula articular.

O diagnóstico histológico foi baseado nos sinais clássicos de uma rede entrelaçada de trabéculas osteoides com grau variável de mineralização, consistente com o nidus, circundadas por osteoblastos e alguns osteoclastos, entremeadas por tecido conjuntivo misto altamente vascularizado
(Figura 7A). A análise do tecido sinovial mostrou edema difuso e infiltrado inflamatório mononuclear perivascular focal. Às vezes, este infiltrado era denso e estava associado à presença de folículos linfoides (Figura 7B). Fibras nervosas focais proeminentes foram encontradas dentro da reação em partes moles adjacentes à lesão em um paciente (Figura 7C).

A intervenção cirúrgica "aberta" com ressecção do nidus foi a técnica utilizada no tratamento dos pacientes, com alívio dos sintomas e/ou melhora funcional em todos os casos. Os pacientes que tiveram melhor resolução do movimento articular foram aqueles cuja duração dos sintomas foi inferior a um ano da admissão. Contudo, o paciente que manifestou sintomas por dois anos também teve melhora significativa após o tratamento cirúrgico.

\section{DISCUSSÃO}

O osteoma osteoide é uma lesão óssea relativamente comum, correspondendo a 10-12\% dos tumores ósseos benignos ${ }^{(7,8)}$. Acomete geralmente indivíduos jovens, na faixa etária de 10 a 25 anos, sendo que em $90 \%$ dos casos ocorre entre 5 e 30 anos de idade, com média de 21 anos $^{\left({ }^{(9)}\right.}$. Os homens são afetados com frequência maior que as mulheres (2:1 a 4:1)

Embora tenha sido descrito pela primeira vez por Bergstrand em 1930, o termo osteoma osteoide foi introduzido na litera- tura médica em 1935 por Jaffe, que o descreveu como uma entidade patológica específica, baseado no estudo de cinco ca$\operatorname{sos}^{(10)}$.

O tumor pode ocorrer em quase todos os ossos do corpo, mas os ossos longos são o local de origem em mais de $70 \%$ dos casos, sendo o fêmur e a tíbia mais comumente afetados (50-60\%). Nos ossos longos, o osteoma osteoide está usualmente localizado na diáfise ou na metáfise. A localização epifisária e intra-articular é rara ${ }^{(11)}$, embora alguns autores estimem sua prevalência em $10-13 \%{ }^{(6,9)}$, sendo o quadril o local mais comum. Tornozelo, cotovelo, punho e joelho são outros locais menos afetados. A lesão é considerada intra-articular quando ocorre na extremidade dos ossos longos, ao redor ou na articulação, num osso limitado pela cápsula, muito próximo dela e da sinóvia.

Sua etiologia é desconhecida. Alterações citogenéticas, como deleções no cromossomo 22q, têm sido descritas no osteoma osteoide ${ }^{(\mathbf{1 2})}$, mas novos estudos são necessários para vincular definitivamente esta entidade a uma mutação clonal. A ocorrência familiar é extremamente rara. Em um trabalho realizado na nossa Instituição ${ }^{(3)}$, o osteoma osteoide foi descrito em dois irmãos que tinham a mesma idade quando os sintomas os levaram a procurar atendimento médico. Ambos também tinham a lesão localizada no córtex do fêmur proximal (local mais comum). Esses acha- 


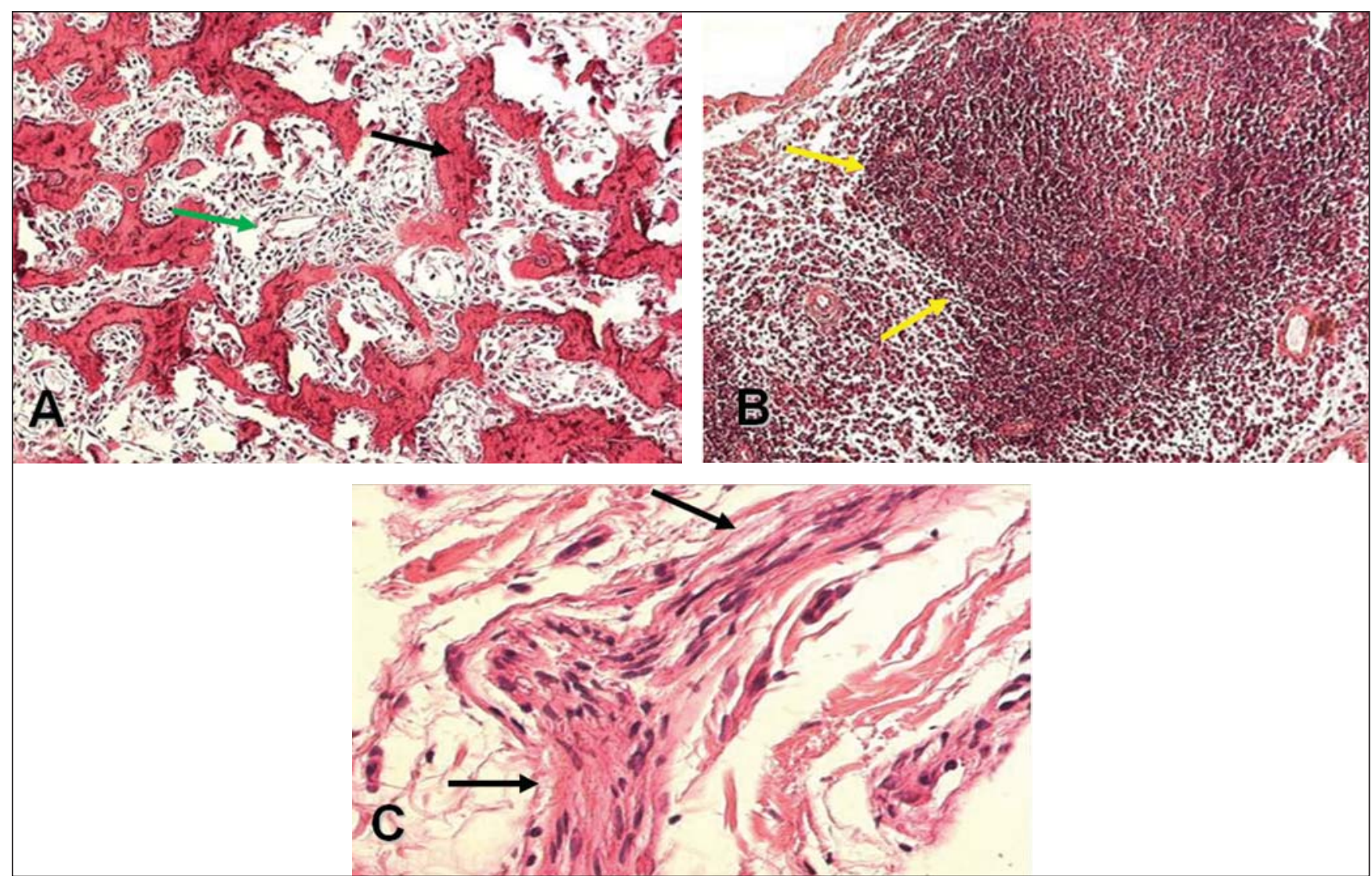

Figura 7. Cortes histológicos. A: Nidus. Rede entrelaçada de trabéculas osteoides (seta preta) permeada por tecido conjuntivo frouxo ricamente vascularizado (seta verde) - coloração hematoxilina-eosina, aumento 4×. B: Sinovite folicular. Tecido sinovial contendo denso infiltrado inflamatório mononuclear, com formação de folículos linfoides (setas) - coloração hematoxilina-eosina, aumento 10×. C: Nervo periférico proeminente (setas) nas partes moles adjacentes ao nidus de osteoma osteoide - coloração hematoxilina-eosina, aumento 10×.

dos sugerem a presença de um fator genético associado.

Estudos imuno-histoquímicos para antígenos neurais e associados a nervos demonstraram que o osteoma osteoide possui fibras nervosas na zona reativa. Sua inervação parece única entre os tumores ós$\operatorname{seos}^{(13)}$. A clínica de dor intensa na área afetada, que piora à noite e melhora em geral dentro de 30 minutos com o uso de salicilatos, está presente em $75 \%$ dos casos e sugere o diagnóstico. A dor parece ser mediada pela liberação de altos níveis de prostaglandinas E2 pelo tumor, que são inibidas pelo uso de anti-inflamatórios não hormonais. As fibras nervosas focais proeminentes encontradas dentro da reação em partes moles adjacentes à lesão em um paciente podem estar relacionadas à dor desproporcional relatada por ele. Isto está em concordância com a descrição anterior de nervos encontrados no tecido peritumoral do osteoma osteoide. Na forma intra-arti- cular, a resposta ao uso de anti-inflamatórios não esteroides no alívio da dor parece ser menos eficaz do que na forma extraarticular. Este dado foi observado em dois dos três pacientes do nosso estudo que utilizaram esse tipo de medicação.

$\mathrm{O}$ tumor tem potencial de crescimento limitado e tendência à maturação espontânea em dois a cinco anos, quando a dor tende a diminuir. A lesão pode regredir espontaneamente, mas o mecanismo desta involução ainda não é conhecido.

A radiografia simples é o exame utilizado na avaliação inicial. A aparência radiográfica clássica do osteoma osteoide consiste em uma lesão lítica redonda ou ovalada, denominada nidus ou nicho, menor que $2,0 \mathrm{~cm}$, normalmente mede de $5 \mathrm{a}$ $10 \mathrm{~mm}$, acompanhada de uma zona de esclerose óssea e/ou espessamento cortical reacional ${ }^{(14)}$. Este aspecto pode variar de acordo com o osso afetado, a localização no osso, a duração dos sintomas e a idade do paciente. O nidus pode ser uniformemente radioluzente ou conter uma quantidade variável de calcificação/ossificação. A maturação óssea é mais acentuada nas lesões com mais tempo de evolução.

O diagnóstico não é difícil quando os achados clínicos e radiográficos típicos estão presentes. Contudo, a apresentação em locais não usuais, como na forma intraarticular, pode ser acompanhada de manifestações atípicas que comprometem o diagnóstico.

O osteoma osteoide pode ser classificado em três tipos: cortical, medular e subperiosteal. Aqueles encontrados em localização intra-articular normalmente são do tipo medular e subperiosteal, o que contribui para uma esclerose limitada ou ausente ao redor do nidus, dificultando sua identificação nas radiografias.

A tomografia com cortes finos $(0,5$ a 2,0 $\mathrm{mm})$ e reconstruções multiplanares utilizando janela óssea é o método de escolha 
para confirmar a natureza benigna da reação óssea e localizar o nidus, principalmente quando este está oculto nas radiografias (Figura 8). A TC também é bastante útil em caso de suspeita de lesão residual. Todavia, esta técnica possui o inconveniente de expor os pacientes à radiação ionizante.

O papel da RM na avaliação do osteoma osteoide é controverso. A aparência variável da lesão, as alterações reacionais extensas na medular óssea e em partes moles podem simular uma lesão mais agressiva ou de outra natureza ${ }^{(15)}$, embora essa não tenha sido a impressão dos autores nos três exames realizados. Baseado na alta vascularização do nidus, o estudo dinâmico com gadolínio combinado com imagens de alta resolução aumenta a conspicuidade do ni$d u s$, sobretudo quando em localização atípica e naqueles pacientes com sintomas clínicos inespecíficos ${ }^{(\mathbf{1 6}-18)}$.

A cintilografia óssea utilizando tecnécio-99 possui alta sensibilidade no diag-

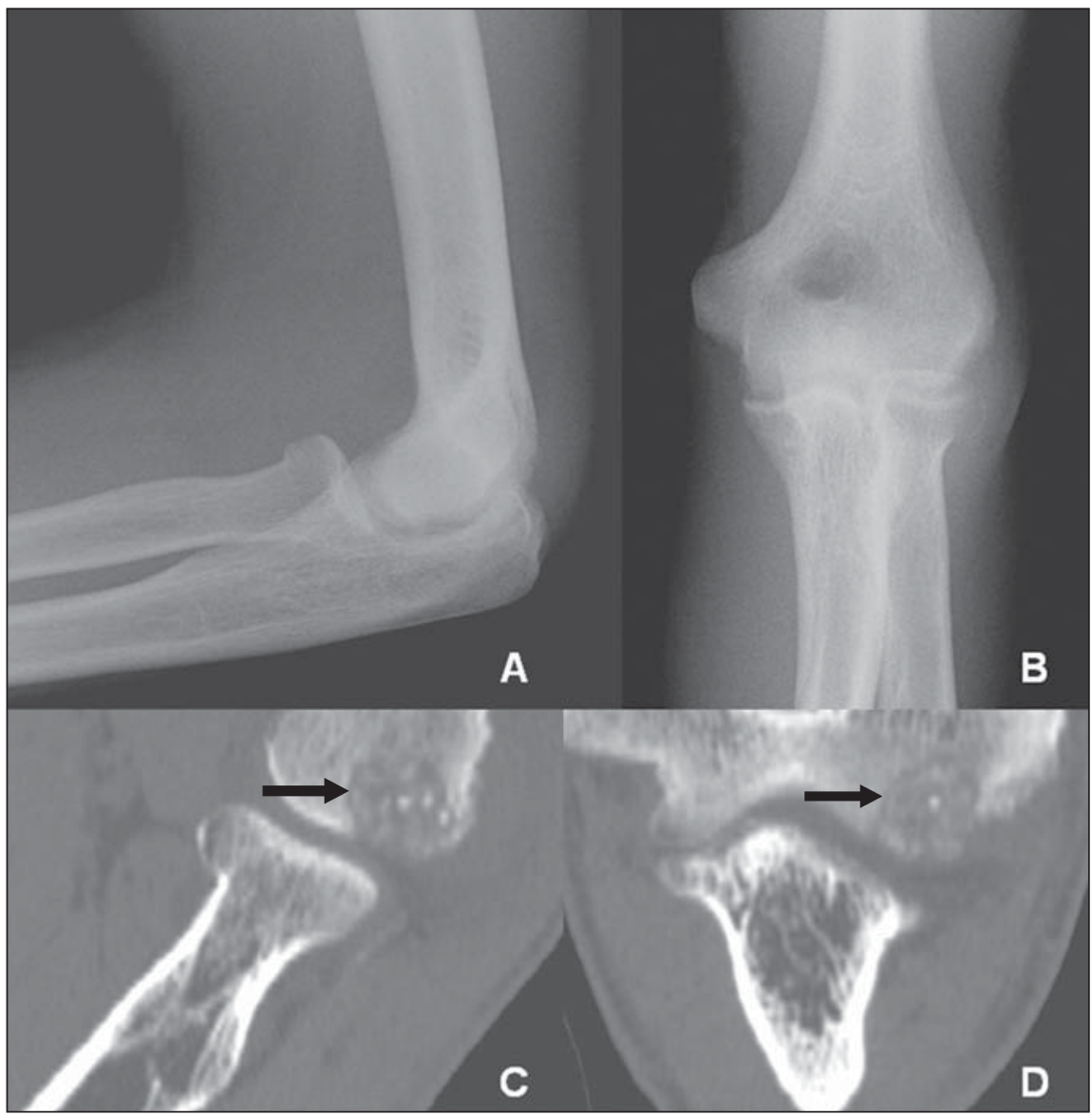

Figura 8. Caso 1. A,B: Radiografias nas incidências lateral e anteroposterior do cotovelo esquerdo não conseguem demonstrar o nidus. C,D: Reconstruções tomográficas nos planos sagital e coronal do mesmo paciente demonstram facilmente a lesão (setas).

nóstico do osteoma osteoide. O tumor possui um padrão típico conhecido como sinal de "dupla densidade". Este sinal é caracterizado por intensa atividade/hipercaptação central na região do nidus e acúmulo menos intenso do radioisótopo na periferia, onde se encontra osso esclerótico reacional e edema perilesional. No entanto, quando a lesão é intra-articular, muitas vezes esse sinal está ausente, pois ocorre um aumento generalizado da atividade dentro da articulação, devido a sinovite associada a osteoporose e hiperemia.

Existem poucos casos de osteoma osteoide no cotovelo descritos na literatura, onde são citados na cabeça e na tuberosidade do rádio, no olécrano e no processo coronoide da ulna, no capítulo e na fossa do olécrano no úmero.

A lesão intra-articular está associada a dor, edema de partes moles e derrame articular. A sinovite pode ser intensa e o paciente apresentar rigidez articular. Dos sete pacientes, seis apresentaram derrame articular. Osteopenia, redução uniforme do espaço articular e alterações osteo-hipertróficas podem ser observadas ${ }^{(9)}$. Clinicamente e radiologicamente, pode simular um processo articular primário, resultando em atraso no diagnóstico por meses ou anos. Pacientes com lesão intra-articular podem evoluir com sinais de osteoartrose secundária à sinovite e proliferação sinovial com destruição da cartilagem. Atrofia muscular pode afetar o membro, especialmente quando o tumor é de longa duração.

O diagnóstico diferencial do osteoma osteoide no cotovelo inclui: monoartrite (inflamatória/infecciosa), sinovite inespecífica, tendinite/epicondilite, osteocondrite dissecante, osteomielite/abscesso de Brodie, granuloma eosinofílico, enostose, fratura de estresse, condroblastoma e osteoblastoma. O osteoma osteoide e o osteoblastoma possuem as mesmas características histopatológicas, porém o osteoblastoma é maior que $2,0 \mathrm{~cm}$, exibe progressão de crescimento e tende a ser mais agressivo.

Nossos resultados, em concordância com estudos anteriores ${ }^{(\mathbf{5})}$, mostraram que os achados radiográficos de esclerose, espessamento cortical e reação periosteal sutil nos ossos do cotovelo, associados à presença de derrame articular, devem levantar a possibilidade de osteoma osteoide.

A excisão completa do nidus é curativa e o tratamento mais tradicional. Entretanto, técnicas minimamente invasivas têm sido utilizadas como alternativas igualmente seguras e eficazes no tratamento do osteoma osteoide, com baixa taxa de morbidade e recuperação mais rápida. A ablação por radiofrequência é a mais proeminente dessas técnicas; por intermédio de uma agulhaeletrodo posicionada no nidus, é transmitida uma corrente alternada de radiofrequência que produz calor e provoca dano celular irreversível/necrose por ação térmica de coagulação ${ }^{(19)}$. Optamos pela intervenção cirúrgica "aberta” para o alívio dos sintomas e melhora funcional dos nossos pacientes. Essa escolha se baseou na dificuldade de diagnóstico pré-operatório, nas hipóteses levantadas, bem como na experiência do cirurgião. Destacamos também a escassez de casos de osteoma osteoide no cotovelo descritos na literatura que nos direcione para uma modalidade de tratamento espe- 
cífica e que possa ser assumida como padrão no tratamento.

\section{CONCLUSÃO}

O osteoma osteoide é uma causa rara de dor no cotovelo. O diagnóstico é difícil, pois as manifestações clínicas e os achados radiográficos geralmente não são característicos. É importante considerar a possibilidade de osteoma osteoide em paciente adulto jovem com dor, limitação do movimento e sinais de sinovite no cotovelo, de etiologia desconhecida, refratária ao tratamento conservador e não relacionada à doença sistêmica. A TC é o exame de escolha para a identificação do nidus. O diagnóstico precoce possibilita o tratamento cirúrgico adequado, diminuindo a morbidade.

\section{Agradecimentos}

À Rede Sarah de Hospitais de Reabilitação, pelo apoio institucional. Aos doutores Antônio Lopes da Cunha Júnior, Sandra Alvarenga Coutinho Passos, Maria Henriqueta Freire Lyra, Jorge Henrique Calil e ao técnico em histologia Cleides
Campos de Oliveira, por suas contribuições neste trabalho.

\section{REFERÊNCIAS}

1. Fletcher CDM, Unni KK, Mertens F, editors. World Health Organization Classification of Tumours. Pathology \& genetics. Tumours of soft tissue and bone. Lyon, France: IARC Press; 2002.

2. Picci P, Mirra JM. Osteoid osteoma. In: Mirra JM, editor. Bone tumors. Philadelphia, PA: Lea \& Febiger; 1989. p. 226-48.

3. Kalil RK, Antunes JS. Familial occurrence of osteoid osteoma. Skeletal Radiol. 2003;32:416-9.

4. Weber KL, Morrey BF. Osteoid osteoma of the elbow: a diagnostic challenge. J Bone Joint Surg Am. 1999;81:1111-9.

5. Moser RP Jr, Kransdorf MJ, Brower AC, et al. Osteoid osteoma of the elbow. A review of six cases. Skeletal Radiol. 1990;19:181-6.

6. Szendroi M, Köllo K, Antal I, et al. Intraarticular osteoid osteoma: clinical features, imaging results, and comparison with extraarticular localization. J Rheumatol. 2004;31:957-64.

7. White ML, Kandel R. Osteoid-producing tumors of bone. Semin Musculoskelet Radiol. 2000;4: 25-43.

8. Dahlin DC, Unni KK. Bone tumors: general aspects and data on 8,542 cases. 4 th ed. Springfield, IL: Thomas; 1987.

9. Allen SD, Saifuddin A. Imaging of intra-articular osteoid osteoma. Clin Radiol. 2003;58:845-52.

10. Jaffe HL. "Osteoid osteoma". A benign osteoblastic tumor composed of osteoid and atypical bone. Arch Surg. 1935;31:709-28

11. Resnick D, Kyriakos M, Greenway GD. Tumors and tumor-like lesions of bone: imaging and pathology of specific lesions. In: Resnick D, Kransdorf MJ, editors. Bone and joint imaging. Philadelphia, PA: Elsevier/Saunders; 2005. p. 1120-6.

12. Baruffi MR, Volpon JB, Barbieri Neto J, et al. Osteoid osteomas with chromosome alterations involving 22q. Cancer Genet Cytogenet. 2001; 124:127-31.

13. O'Connell JX, Nanthakumar SS, Nielsen GP, et al. Osteoid osteoma: the uniquely innervated bone tumor. Mod Pathol. 1998;11:175-80.

14. Cassard X, Accadbled F, De Gauzy JS, et al. Osteoid osteoma of the elbow in children: a report of three cases and a review of the literature. J Pediatr Orthop B. 2002;11:240-4.

15. Davies M, Cassar-Pullicino VN, Davies AM, et al. The diagnostic accuracy of MR imaging in osteoid osteoma. Skeletal Radiol. 2002;31:559-69.

16. Liu PT, Chivers FS, Roberts CC, et al. Imaging of osteoid osteoma with dynamic gadolinium-enhanced MR imaging. Radiology. 2003;227:691700 .

17. Zampa V, Bargellini I, Ortori S, et al. Osteoid osteoma in atypical locations: the added value of dynamic gadolinium-enhanced MR imaging. Eur J Radiol. 2009;71:527-35.

18. von Kalle T, Langendörfer M, Fernandez FF, et al. Combined dynamic contrast-enhancement and serial 3D-subtraction analysis in magnetic resonance imaging of osteoid osteomas. Eur Radiol. 2009;19:2508-17.

19. Motamedi D, Learch TJ, Ishimitsu DN, et al Thermal ablation of osteoid osteoma: overview and step-by-step guide. Radiographics. 2009;29: 2127-41. 